\title{
MODULES THAT HAVE A WEAK SUPPLEMENT IN EVERY EXTENSION
}

\author{
EMINE ÖNAL, HAMZA ÇALIŞICI, AND ERGÜL TÜRKMEN
}

Received 13 November, 2014

\begin{abstract}
We say that over an arbitrary ring a module $M$ has the property $(W E$ ) (respectively, $(W E E))$ if $M$ has a weak supplement (respectively, ample weak supplements) in every extension. In this paper, we provide various properties of modules with these properties. We show that a module $M$ has the property (WEE) iff every submodule of $M$ has the property $(W E)$. A ring $R$ is left perfect iff every left $R$-module has the property (WE) iff every left $R$-module has the property (WEE). A ring $R$ is semilocal iff every left $R$-module has a weak supplement in every extension with small radical. We also study modules that have a weak supplement(respectively, ample weak supplements) in every coatomic extension, namely the property $\left(W E^{*}\right)$ (respectively, $\left.\left(W E E^{*}\right)\right)$.
\end{abstract}

2010 Mathematics Subject Classification: 16D10; 16L30

Keywords: weak supplement, coatomic extension, semilocal ring, left perfect ring

\section{INTRODUCTION}

Throughout this paper, $R$ is an associative ring with identity and all modules are unital left $R$-modules, unless otherwise stated. Let $M$ be an $R$-module. The notation $U \leq M$ means that $U$ is a submodule of $M$. A submodule $U$ of $M$ is called small in $M$, denoted as $U<<M$, if $M \neq U+L$ for every proper submodule $L$ of $M$. By $\operatorname{Rad}(M)$ we denote the intersection of all maximal submodules of $M$, equivalently the sum of all small submodules of $M$ (see [14]). A module $M$ is called radical if $M$ has no maximal submodules, that is, $M=\operatorname{Rad}(M)$.

As a proper generalization of direct summands of a module, the notion of supplement submodules is defined. For $U, V$ submodules of a module $M, V$ is called a supplement of $U$ in $M$ if it is minimal with respect to $M=U+V$, equivalently $M=U+V$ and $U \cap V \ll V$. Then, it is natural to introduce a generalization of supplement submodules by [14, Section 19.3.(2)]. A submodule $V$ of $M$ is called a weak supplement of $U$ in $M$ if $U+V=M$ and $U \cap V \ll M$. A module $M$ is called weakly supplemented if every submodule of $M$ has a weak supplement in $M$ (see [9], [14] and [17]). A submodule $U$ of $M$ has ample (weak) supplements in $M$ 
if, whenever $M=U+L, L$ contains a (weak) supplement of $U$ in $M$. Under given definitions, we clearly have the following implication on submodules:

$$
\text { direct summand } \Longrightarrow \text { supplement } \Longrightarrow \text { weak supplement }
$$

Let $R$ be a ring and $M$ be an $R$-module. An $R$-module $N$ is called an extension of $M$ provided $M \subseteq N$. A module $M$ is said to be injective if it is a direct summand in its every extension $N$.

Modules that have a supplement (resp. ample supplements) in every extension, i.e. modules with the property $(E)$ (resp. $(E E)$ ), was first introduced by $\mathrm{H}$. Zöschinger in [16], as a generalization of injective modules. The author determined in the same paper the structure of modules with these properties.

Adapting his concepts, we introduce the properties $(W E)$ and $(W E E)$ as a generalization of the properties $(E)$ and $(E E)$ in Section 2. We call a module that has the property $(W E)$ (resp. $(W E E)$ ) if it has a weak supplement (resp. ample weak supplements) in every extension. Moreover in this section, we show that a module $M$ has the property $(W E E)$ if and only if every submodule of $M$ has the property $(W E)$. This gives us that every submodule of a module with the property (WEE) is weakly supplemented. We prove that the property $(W E)$ is inherited by direct summands. In Corollary 2, we obtain that if a ring $R$ is left hereditary, then every factor module of an R-module with the property $(W E)$ has the property $(W E)$. Thanks to Lemma 3.3 of Zöschinger's paper [16], we directly say that over a complete local dedekind domain $R$, an $R$-module $M$ has the property ( $W E$ ) if and only if $M$ has the property $(E)$. We also give new characterizations of left perfect rings via the modules with the properties $(W E)$ and $(W E E)$.

Let $R$ be a ring and $M$ be an $R$-module. R. Alizade et al. [1] say a submodule $U$ of $M$ cofinite in $M$ if the factor module $\frac{M}{U}$ is finitely generated. In [5], H. Çalışıcı and E. Türkmen called an extension $N$ of $M$ cofinite extension if $M$ is cofinite in $N$. Following [5], the authors studied modules that have a supplement (resp. ample supplements) in every cofinite extension, namely the property $(C E)$ (resp. $(C E E))$, as a generalization of the property $(E)$ (resp. $(E E)$ ). In addition, they showed in [5, Theorem 2.12] that a ring $R$ is semiperfect if and only if every left $R$-module has the property $(C E)$.

In [15], a module $M$ is said to be coatomic if $\operatorname{Rad}\left(\frac{M}{K}\right)=\frac{M}{K}$ implies that $K=M$ for some submodule $K$ of $M$, that is, every radical factor module of $M$ is zero. $M$ is coatomic if and only if every proper submodule of $M$ is contained in a maximal submodule of $M$. Note that semisimple modules are coatomic.

Let $R$ be a ring and $M, N$ be $R$-modules. $N$ is called a coatomic extension of $M$ in case $M \subseteq N$ and $\frac{N}{M}$ is coatomic. In [11], B. N. Türkmen studied on modules that have a supplement (resp. ample supplements) in every coatomic extension and termed these modules $E^{*}$-modules (resp. $E E^{*}$-modules). Since finitely generated modules are coatomic, $E^{*}$-modules (resp. $E E^{*}$-modules) have the property $(C E)$ (resp. $(C E E))$. 
In Section 3, we also call a module that has the property $\left(W E^{*}\right)\left(\right.$ resp. $\left(W E E^{*}\right)$ ) if it has a weak supplement (resp. ample weak supplements) in every coatomic extension. We prove that over a left $V$-ring $R$, every left $R$-module with $\left(W E^{*}\right)$ is injective. In addition, we give also a characterization of semilocal rings via the modules that have a weak supplement in every extension with small radical. Finally, we give an example of modules that have a weak supplement in every extension with small radical but not have the property $(C E E)$.

\section{Modules With the PRoperties (WE) AND ( $W E E)$}

It is shown in [16, Lemma 1.3.(a)] that direct summands of modules with the property $(E)$ have the property $(E)$. Now we give an analogue of this fact for the modules with the property $(W E)$.

Proposition 1. Let $M$ be a module. If $M$ has the property $(W E)$, then every direct summand of $M$ has the property (WE).

Proof. Let $M_{1}$ be a direct summand of $M$. Then there exists a submodule $M_{2}$ of $M$ such that $M=M_{1} \oplus M_{2}$. Let $N$ be any extension of $M_{1}$. Let $N^{\prime}$ be the external direct sum $N \oplus M_{2}$ and $\vartheta: M \rightarrow N^{\prime}$ be the canonical embedding. Then $M \cong \vartheta(M)$ has the property $(W E)$. Hence, there exists a submodule $V$ of $N^{\prime}$ such that $N^{\prime}=\vartheta(M)+V$ and $\vartheta(M) \cap V \ll N^{\prime}$. By the projection $\pi: N^{\prime} \rightarrow N$, we have that $M_{1}+\pi(V)=N$. Also since $\operatorname{Ker}(\pi) \subseteq \vartheta(M), \pi(\vartheta(M) \cap V)=\pi(\vartheta(M)) \cap$ $\pi(V)=M_{1} \cap \pi(V) \ll N$. Hence $\pi(V)$ is a weak supplement of $M_{1}$ in $N$.

Proposition 2. A module $M$ has the property (WEE) if and only if every submodule of $M$ has the property (WE).

Proof. Suppose that every submodule of $M$ has the property $(W E)$. For any extension $N$ of $M$, let $N=M+K$ for some submodule $K$ of $N$. Since $M \cap K$ has the property $(W E)$, there exists a submodule $L$ of $K$ such that $(M \cap K)+L=K$ and $(M \cap K) \cap L=M \cap L \ll K$. Note that $N=M+K=M+((M \cap K)+L))=$ $M+L$. It follows that $L$ is a weak supplement of $M$ in $N$.

Conversely, let $M$ be a module with the property $(W E E)$ and $M_{1}$ be any submodule of $M$. For any extension $N$ of $M_{1}$, let $F=\frac{M \oplus N}{H}$, where the submodule $H$ is the set of all elements $\left(m^{\prime},-m^{\prime}\right)$ of $M \oplus N$ with $m^{\prime} \in M_{1}$ and let $\gamma: M \rightarrow F$ via $\gamma(m)=(m, 0)+H, \psi: N \rightarrow F$ via $\psi(n)=(0, n)+H$ for all $m \in M, n \in N$. For inclusion homomorphisms $\iota_{1}: M_{1} \rightarrow N$ and $\iota_{2}: M_{1} \rightarrow M$, we can draw the following pushout:

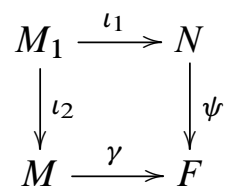


It is clear that $F=\operatorname{Im}(\gamma)+\operatorname{Im}(\psi)$. Since $\gamma$ is monomorphism, by assumption, $\operatorname{Im}(\gamma)$ has the property $(W E E)$. It means that $\operatorname{Im}(\gamma)$ has a weak supplement $V$ in $F$ such that $V \leq \operatorname{Im}(\psi)$, i.e. $F=\operatorname{Im}(\gamma)+V$ and $\operatorname{Im}(\gamma) \cap V \ll F$. Then we obtain that $N=\psi^{-1}(\operatorname{Im}(\gamma))+\psi^{-1}(V)=M_{1}+\psi^{-1}(V)$ and $M_{1} \cap \psi^{-1}(V) \ll N$. Hence $\psi^{-1}(V)$ is a weak supplement of $M_{1}$ in $N$.

Corollary 1. Every submodule of a module with the property (WEE) is weakly supplemented.

Lemma 1. Every simple submodule $S$ of a module $M$ is either a direct summand of $M$ or small in $M$.

Proof. Suppose that $S$ is not small in $M$, then there exists a proper submodule $K$ of $M$ such that $S+K=M$. Since $S$ is simple and $K \neq M, S \cap K=0$. Thus $M=S \oplus K$.

Let $R$ be a ring and $M$ be an $R$-module. $M$ is called local if the sum of all proper submodules of $M$ is a proper submodule of $M$. $R$ is called a local ring if $R R$ (or $\left.R_{R}\right)$ is a local module.

Proposition 3. Local modules have the property (WE).

Proof. Let $S$ be a module and $N$ be any extension of $S$. If $S$ is small in $N, N$ is a weak supplement of $S$ in $N$. Suppose that $S$ is not small in $N$. Then there is a proper submodule $S^{\prime}$ of $N$ such that $S+S^{\prime}=N$. From Lemma 1, if $S$ is simple, $S^{\prime}$ is a direct summand of $N$. If $S$ is local, $S \cap S^{\prime}$ is small in $S$. In both cases, $S^{\prime}$ is a weak supplement of $S$ in $N$.

Let $M$ be a module and $U$ be a submodule of $M$. If the factor module $\frac{M}{U}$ has the property $(W E), M$ does not need to have the property $(W E)$. For example, for the ring $R=\mathbb{Z}$, the $R$-module $M=\frac{2 \mathbb{Z}}{6 \mathbb{Z}}$ has a weak supplement in every extension because it is simple. But $2 \mathbb{Z}$ does not have a weak supplement in its extension $\mathbb{Z}$. Now we show that the statement mentioned above is true under a special condition.

Proposition 4. Let $M$ be a module and $U$ be a submodule of $M$. If $U \ll M$ and the factor module $\frac{M}{U}$ has the property $(W E), M$ has the property $(W E)$.

Proof. Let $N$ be any extension of $M$. Since $\frac{M}{U}$ has the property $(W E)$, there exists a submodule $\frac{V}{U}$ of $\frac{N}{U}$ such that $\frac{M}{U}+\frac{V}{U}=\frac{N}{U}$ and $\frac{M \cap V}{U} \ll \frac{N}{U}$. Note that $M+V=N$. Suppose that $M \cap V+S=N$ for a submodule $S$ of $N$. Then we obtain $\frac{M \cap V}{U}+\frac{S+U}{U}=\frac{N}{U}$. Since $\frac{M \cap V}{U} \ll \frac{N}{U}$, we have that $\frac{S+U}{U}=\frac{N}{U}$. By hypothesis, it follows that $N=S+U=S$. Hence $M \cap V \ll N$.

For a module $M$, we will denote by $\operatorname{Soc}(M)$ the sum of all simple submodules of $M$. Note that $\operatorname{Soc}(M)$ is the largest semisimple submodule of $M$. 
Remark 1. Let $M$ be a finitely generated semisimple module. Then $M$ is artinian. Since artinian modules have the property $(E)$, it has the property $(W E)$. Note that here the condition "finitely generated" is necessary. For example, consider the left $\mathbb{Z}$-module $M=\prod_{p \in \Omega} \frac{\mathbb{Z}}{p \mathbb{Z}}$, where $\Omega$ is the set of all prime numbers. Then, the semisimple module $\operatorname{Soc}(M)=\bigoplus_{p \in \Omega} \frac{\mathbb{Z}}{p \mathbb{Z}}$. By [3, Lemma 2.9], there exists a submodule $N$ of $M$ such that $\frac{N}{\operatorname{Soc}(M)} \cong \mathbb{Q}$. If $\operatorname{Soc}(M)$ has a weak supplement $K$ in $N$, we have $N=\operatorname{Soc}(M) \oplus K$ since $\operatorname{Rad}(M)=0$. Therefore, $K$ is injective and so $K=\operatorname{Rad}(K) \subseteq \operatorname{Rad}(M)=0$, a contradiction.

In [7] a ring $R$ is said to be a left $V$-ring if every simple left $R$-module is injective. It is well known that a ring $R$ is a left $V$-ring if and only if $\operatorname{Rad}(M)=0$ for every left $R$-module $M$. A ring $R$ is called left hereditary if every left ideal of $R$ is projective. $R$ is a left hereditary ring if and only if every factor module of an injective left $R$ module is injective [14, Section 39.16].

The next example shows that every factor module of a module with the property $(W E)$ does not need to have the property $(W E)$. Firstly we need the following lemma.

Lemma 2. Let $R$ be a left $V$-ring. An $R$-module $M$ has the property $(W E)$ if and only if $M$ is injective.

Proof. Let $M$ has the property $(W E)$ and $N$ be any extension of $M$. Then $M$ has a weak supplement $V$ in $N$. We have $M+V=N, M \cap V \ll N$. Hence $M \cap V \leqslant$ $\operatorname{Rad}(N)$. Since $\operatorname{Rad}(N)=0$, we have $N=M \oplus V$.

Conversely, let $M$ be injective and $N$ be any extension of $M$. Then there exists a submodule $K$ of $N$ such that $N=M \oplus K$. Hence $K$ is a weak supplement of $M$ in $N$.

Example 1. Let $R$ be the product of the family $\left\{F_{i}\right\}_{i \in I}$, where each $F_{i}$ is a field for an infinite index set $I$. The ring $R$ is a commutative Von Neumann regular but not hereditary [10, Example 2.15]. Then by [14, Section 23.5], $R$ is a left $V$-ring. $R$ is injective from [8, Corollary, 3.11.B]. By Lemma 2, the left $R$-module ${ }_{R} R$ has the property (WE). Since $R$ is not hereditary, there is at least one factor module of $R$ which is not injective. This factor module does not have the property $(W E)$ by using Lemma 2.

Next we prove that under proper conditions a factor module of a module with the property $(W E)$ has the property $(W E)$.

Proposition 5. Let $K \subseteq M \subseteq L$ be modules with $\frac{L}{K}$ injective. If $M$ has the property $(W E)$, then $\frac{M}{K}$ has the property $(W E)$.

Proof. Let $N$ be any extension of $\frac{M}{K}$. Since $\frac{L}{K}$ is injective, by [10, Lemma 2.16] we have the following commutative diagram with exact rows: 


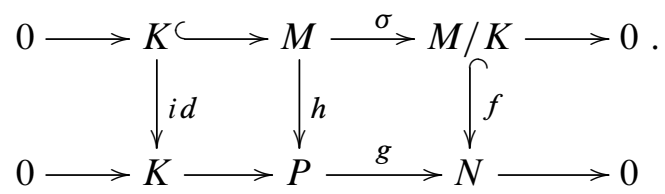

Since $h$ is monomorphism and $M$ has the property $(W E), M \cong I m(h)$ has a weak supplement $V$ in $P$, that is, $\operatorname{Im}(h)+V=P$ and $\operatorname{Im}(h) \cap V \ll P$. We claim that $g(V)$ is a weak supplement of $\frac{M}{K}$ in $N$.

$N=g(P)=g(h(M))+g(V)=(f \sigma)(M)+g(V)=\frac{M}{K}+g(V)$ and $\frac{M}{K} \cap g(V)=f(\sigma(M)) \cap g(V)=g[h(M) \cap V] \ll g(P)$. Hence $\frac{M}{K} \cap g(V) \ll N$.

Corollary 2. If $R$ is a left hereditary ring and $M$ is an $R$-module with the property $(W E)$, then every factor module of $M$ has the property $(W E)$.

If a module $M$ has a supplement in its injective envelope, $M$ need not to have a weak supplement in every extension. For example, for the ring $R=\mathbb{Z}$, the $R$-module $M=2 \mathbb{Z}$ has a supplement in its injective envelope $\mathbb{Q}$. But $M=2 \mathbb{Z}$ does not have a weak supplement in its extension $\mathbb{Z}$. Now we prove that over a local dedekind domain, a module $M$ has a supplement in its injective envelope if and only if $M$ has a weak supplement in every extension.

Lemma 3. Let $R$ be a local dedekind domain and $M$ be an $R$-module. The following statements are equivalent:

(1) $M$ has a supplement in its injective envelope.

(2) $M$ has the property (WE).

(3) $M$ is an $E^{*}$-module.

Proof. It is clear by [16, Lemma 3.3].

Proposition 6. Let $R$ be a complete local dedekind domain and $M$ be an $R$ module. $M$ has the property (WE) if and only if $M$ has the property $(E)$.

Proof. Let $M$ has the property $(W E)$ and $N$ be any extension of $M$. Since $M$ has the property $(W E)$, there exists a submodule $X$ of $N$ such that $M+X=N$, $M \cap X \ll N$. By [16, Section 3, Corollary 5], there exists a supplement $V$ of $M$ in $N$ with $V \subset X$. Hence $M$ has the property $(E)$.

Proposition 7. Let $R$ be a non-local dedekind domain and $M$ be a semisimple $R$-module. Then, the following three statements are equivalent:

(1) $M$ has the property (WE).

(2) $M$ has the property $(E)$.

(3) $M$ is of the form $K \oplus \prod_{p} A_{p}$, where $K$ is injective and $A_{p}$ is a bounded $p$-primary module for every prime element $p \in R$. 
Proof. (1) $\Longleftrightarrow(2)$ It follows from [12, Proposition 2.1].

(2) $\Longleftrightarrow$ (3) By [16, Theorem 5.6].

It is known from [14, Section 43.9] that a ring $R$ is left perfect if and only if every left $R$-module has the property $(E)$. The next theorem gives new characterizations of left perfect rings via their modules which have the property $(W E)$.

Theorem 1. For a ring $R$ the following statements are equivalent:

(1) $R$ is left perfect.

(2) Every left $R$-module is weakly supplemented.

(3) Every left $R$-module has the property $(W E)$.

(4) $R^{(\mathbb{N})}$ is weakly supplemented.

(5) $R^{(\mathbb{N})}$ has the property $(W E E)$.

(6) Every left $R$-module has the property (WEE).

Proof. (1) $\Leftrightarrow(2) \Leftrightarrow$ (4) is clear from [4, Theorem 1]. (3) $\Rightarrow$ (6) and (5) $\Rightarrow$ (4) follow from Proposition 2. (1) $\Rightarrow$ (3) follows from [14, Section 43.9]. (6) $\Rightarrow(5)$ is clear.

The following definitions are given in the paper [6], and we recall them for the convenience of the reader:

By a valuation ring (also called a chain ring) we mean a commutative ring $R$ whose ideals are totally ordered by inclusion. Equivalently, if $a, b \in R$, then either $a \in R b$ or $b \in R a$. A valuation ring that is a domain will be called a valuation domain. A valuation ring $R$ is called maximal if ${ }_{R} R$ is linearly compact, i.e., every family of cosets $\left\{a_{i}+L_{i} \mid i \in I\right\}$ with the finite intersection property has a non-empty intersection. Since linearly compact modules have ample supplements in every extension, a maximal ring $R$ has the property ( $W E E$ ).

The following example shows that a ring with the property $(W E E)$ need not be left perfect, in general.

Example 2. Let $R$ be the localization ring $\mathbb{Z}_{(p)}$ of the ring $\mathbb{Z}$ of integers at a prime ideal $p \mathbb{Z} \neq 0$. Then, the completion of $\mathbb{Z}_{(p)}$, the ring $J_{(p)}$ of $p$-adic integers, is a maximal valuation domain which is not field. Hence, $J_{(p)}$ has the property $(W E E)$ but not perfect.

\section{Modules With THE PROPERTIES $\left(W E^{*}\right)$ AND $\left(W E E^{*}\right)$}

In this section, we study on modules with the property $\left(W E^{*}\right)$ (resp. $\left.\left(W E E^{*}\right)\right)$, which have a weak supplement (resp. ample weak supplements) in every coatomic extension, as a generalization of modules with the property $(W E)$ (resp. (WEE)). We prove that over a left $V$-ring $R$, every left $R$-module with the property $\left(W E^{*}\right)$ is injective.

Proposition 8. Let $M$ be a module. If $M$ has the property $\left(W E^{*}\right)$, then every direct summand of $M$ has the property $\left(W E^{*}\right)$. 
Proof. Let $M_{1}$ be a direct summand of $M$ and $N$ be a coatomic extension of $M_{1}$. Then there exists a submodule $M_{2}$ of $M$ such that $M=M_{1} \oplus M_{2}$. Let $N^{\prime}$ be the external direct sum $N \oplus M_{2}$ and $\varphi: M \longrightarrow N^{\prime}$ be the canonical embedding. Then $M \cong \varphi(M)$ has the property $\left(W E^{*}\right)$. Note that $\frac{N}{M_{1}} \cong \frac{N \oplus M_{2}}{\varphi(M)}=\frac{N^{\prime}}{\varphi(M)}$ is coatomic. Since $\varphi(M)$ has the property $\left(W E^{*}\right)$, there exists a submodule $V$ of $N^{\prime}$ such that $N^{\prime}=\varphi(M)+V$ and $\varphi(M) \cap V \ll N^{\prime}$. For the projection $\phi: N^{\prime} \longrightarrow N$, we have that $M_{1}+\phi(V)=N$. Also since $\operatorname{Ker}(\phi) \subseteq \varphi(M), \phi(\varphi(M) \cap V) \subseteq \phi(\varphi(M)) \cap \phi(V)=$ $M_{1} \cap \phi(V) \ll \phi\left(N^{\prime}\right)=N$. Hence $\phi(V)$ is a weak supplement of $M_{1}$ in $N$.

Proposition 9. A module $M$ has the property (WE $\left.E^{*}\right)$ if and only if every submodule of $M$ has the property $\left(W E^{*}\right)$.

Proof. Assume that every submodule of $M$ has the property $\left(W E^{*}\right)$. For a coatomic extension $N$ of $M$, let $N=M+V$ for some submodule $V$ of $N$. Then $\frac{N}{M} \cong \frac{V}{M \cap V}$ is coatomic and so $V$ is a coatomic extension of $M \cap V$. Since $M \cap V$ has the property $\left(W E^{*}\right)$, there exists a submodule $K$ of $V$ such that $V=M \cap V+K$ and $M \cap K \ll V$. Note that $N=M+V=M+(M \cap V+K)=M+K$. It follows that $K$ is a weak supplement of $M$ in $N$.

Conversely, let $M$ be a module with the property $\left(W E E^{*}\right)$ and let $M_{1}$ be any submodule of $M$. For a coatomic extension $N$ of $M_{1}$, let $S=\frac{M \oplus N}{L}$, where the submodule $L$ is the set of all elements $\left(m^{\prime},-m^{\prime}\right)$ of $M \oplus N$ with $m^{\prime} \in M_{1}$ and let $f: M \rightarrow S$ via $f(m)=(m, 0)+L, g: N \rightarrow S$ via $g(n)=(0, n)+L$ for all $m \in M, n \in N$. For the inclusion homomorphisms $\tau_{1}: M_{1} \rightarrow N$ and $\tau_{2}: M_{1} \rightarrow M$, we can draw the following pushout:

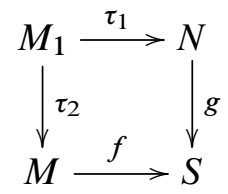

It is clear that $S=\operatorname{Im}(f)+\operatorname{Im}(g)$. Now we define $\theta: S \rightarrow \frac{N}{M_{1}}$ by $\theta((m, n)+L)=$ $n+M_{1}$ for all $(m, n)+L \in S$. Note that $\theta$ is an epimorphism and $\operatorname{Ker}(\theta)=\operatorname{Im}(f)$. It follows that $\frac{N}{M_{1}} \cong \frac{S}{\operatorname{Im}(f)}$ is coatomic. Since $f$ is monomorphism, by assumption, $\operatorname{Im}(f)$ has the property $\left(W E E^{*}\right)$. Then it follows immediately that $\operatorname{Im}(f)$ has a weak supplement $V$ in $S$ such that $V \leq \operatorname{Im}(g)$, i.e. $S=\operatorname{Im}(f)+V$ and $\operatorname{Im}(f) \cap$ $V \ll S$. Then we obtain that $N=g^{-1}(\operatorname{Im}(f))+g^{-1}(V)=M_{1}+g^{-1}(V)$ and $M_{1} \cap g^{-1}(V) \ll N$. Hence $g^{-1}(V)$ is a weak supplement of $M_{1}$ in $N$.

Recall from [2] a module $M$ is called cofinitely weak supplemented if every cofinite submodule of $M$ has a weak supplement in $M$. It is clear from Proposition 9 that if a module $M$ has the property $\left(W E E^{*}\right)$, then every maximal submodule of $M$ has a weak supplement in $M$, equivalently $M$ is cofinitely weak supplemented by [2, Theorem 2.16]. 
In [13], a module $M$ is called weakly radical supplemented (namely wrs-module) if every submodule $U$ of $M$ with $\operatorname{Rad}(M) \subseteq U$ has a weak supplement in $M$. A module $M$ is called semilocal if $\frac{M}{\operatorname{Rad}(M)}$ is semisimple. A ring $R$ is semilocal if the left $R$-module ${ }_{R} R$ is semilocal.

Corollary 3. Let $R$ be a semilocal ring and $M$ be an $R$-module. If $M$ has the property $\left(W E E^{*}\right)$, then $M$ is wrs-module.

Proof. Let $U$ be a submodule of $M$ with $\operatorname{Rad}(M) \subseteq U$. Since $R$ is semilocal ring, it follows from [9, Theorem 3.5] that $\frac{M}{U}$ is semisimple as a factor module of the semisimple module $\frac{M}{\operatorname{Rad}(M)}$. Hence $\frac{M}{U}$ is coatomic. By assumption and Proposition 9, $U$ has a weak supplement in $M$. Hence $M$ is a wrs-module.

Proposition 10. Over a left $V$-ring $R$, every left $R$-module with $\left(W E^{*}\right)$ is injective.

Proof. Let $M$ be an $R$-module with $\left(W E^{*}\right)$. Let $N$ be any extension of $M$. Suppose that $\operatorname{Rad}\left(\frac{N}{K}\right)=\frac{N}{K}$ for a submodule $K$ of $N$. Since $R$ is a left $V$-ring, $\operatorname{Rad}\left(\frac{N}{K}\right)=0$. Then it immediately follows that $N=K$. Hence $N$ is coatomic. Then, by assumption, $M$ has a weak supplement $V$ in $N$, i.e. $N=M+V$ and $M \cap V \ll N$. Since $R$ is a left $V$-ring, we obtain that $M \cap V \subseteq \operatorname{Rad}(N)=0$. This completes the proof.

The next result can be directly obtained from Proposition 10 and Lemma 2.

Corollary 4. Let $R$ be a left $V$-ring and $M$ be an $R$-module. The following statements are equivalent:

(1) $M$ has the property (WE).

(2) $M$ has the property $\left(W E^{*}\right)$.

(3) $M$ is injective.

Now we shall give a characterization for semilocal rings via the modules that have a weak supplement in every extension with small radical.

Theorem 2. For any ring $R$ the following statements are equivalent:

(1) $R$ is semilocal.

(2) Every left $R$-module with small radical is weakly supplemented.

(3) Every left $R$-module has a weak supplement in every extension with small radical.

Proof. (1) $\Leftrightarrow$ (2) follows from [9, Theorem 3.5].

(2) $\Leftrightarrow$ (3) $M$ be a left $R$-module and $N$ be an extension of $M$ with small radical. By hypothesis, $M$ has a weak supplement in $N$. Conversely, let $M$ be an $R$-module with small radical and $U$ be a submodule of $M$. By assumption, $U$ has a weak supplement in $M$. 
Finally, we give an example of modules that have a weak supplement in every extension with small radical but not have the property $(C E E)$.

Example 3. (see [14, Section 42.13, Exercise 4]). Let $R$ be the following subring of the rational numbers:

$R=\left\{\frac{m}{n} \mid m, n \in \mathbb{Z},(m, n)=1,2\right.$ and 3 are not divisors of $\left.n\right\}$

Since $\frac{R}{\operatorname{Rad}(R)}$ is semisimple, the left $R$-module ${ }_{R} R$ is a module which has a weak supplement in every extension with small radical by Theorem 2 . Whereas, since $R$ is not semiperfect, ${ }_{R} R$ does not have the property $(C E E)$ by [5, Theorem 2.12].

\section{ACKNOWLEDGEMENT}

The authors would like to thank the referee for many valuable suggestions and comments in the revision of this paper.

\section{REFERENCES}

[1] R. Alizade, G. Bilhan, and P. Smith, "Modules whose maximal submodules have supplements," Comm. Algebra, vol. 29, no. 6, pp. 2389-2405, 2001, doi: 10.1081/AGB-100002396.

[2] R. Alizade and E. Büyükaşık, "Cofinitely weak supplemented modules," Comm. Algebra, vol. 31, no. 11, pp. 5377-5390, 2003.

[3] R. Alizade and E. Büyükaşık, "Extensions of weakly supplemented modules," Math. Scand., vol. 103, no. 2, pp. 161-168, 2008.

[4] E. Büyükaşik and C. Lomp, "Rings whose modules are weakly supplemented are perfect. Applications to certain ring extensions," Math. Scand., vol. 105, pp. 25-30, 2009.

[5] H. Çalışıcı and E. Türkmen, "Modules that have a supplement in every cofinite extension," Georgian Math. J., vol. 19, no. 2, pp. 209-216, 2012, doi: 10.1515/gmj-2012-0018.

[6] L. Fuchs and S. L., Modules over Non-Noetherian Domains, ser. Math. Surveys Monographs. American Mathematical Society, Providence, 2000.

[7] S. K. Jain, A. K. Srivastava, and A. A. Tuganbaev, Cyclic modules and the structure of rings, ser. Oxford Mathematical Monographs. Oxford University Press, Oxford, 2012. doi: 10.1093/acprof:oso/9780199664511.001.0001.

[8] T. Lam, Lectures on Modules and Rings, ser. Graduate texts in mathematics. Springer New York, 1999. doi: 10.1007/978-1-4612-0525-8.

[9] C. Lomp, "On semilocal modules and rings," Comm. Algebra, vol. 27, no. 4, pp. 1921-1935, 1999.

[10] S. Özdemir, "Rad-supplementing modules," arXiv: 1210.2893v1 [math.RA], 10 Oct. 2012.

[11] B. N. Türkmen, "Modules that have a supplement in every coatomic extension," Miskolc Mathematical Notes, vol. 16, no. 1, pp. 543-551, 2015.

[12] B. N. Türkmen, "On generalizations of injective modules," Publications de l'Institut Mathematique, (accepted).

[13] B. N. Türkmen and E. Türkmen, "On a generalization of weakly supplemented modules," Analele Stiintifice ale Universitatii "Al. I. Cuza" din Iasi, Matematica, 2015, doi: 10.1515/aicu-20150012.

[14] R. Wisbauer, Foundations of module and ring theory, ser. Algebra, Logic and Applications. Gordon and Breach Science Publishers, Philadelphia, PA, 1991, vol. 3, a handbook for study and research.

[15] H. Zöschinger, “Komplementierte Moduln über Dedekindringen,” J. Algebra, vol. 29, pp. 42-56, 1974, doi: 10.1016/0021-8693(74)90109-4. 
[16] H. Zöschinger, "Moduln, die in jeder Erweiterung ein Komplement haben,” Math. Scand., vol. 35, pp. 267-287, 1974.

[17] H. Zöschinger, "Invarianten wesentlicher Überdeckungen,” Math. Annalen, vol. 237, no. 3, pp. 193-202, 1978, doi: 10.1007/BF01420175.

Authors' addresses

Emine Önal

Ahi Evran University, Faculty of Art and Science, Department of Mathematics, Bağbaşi/Kirşehir, Turkey

E-mail address: emine.onaleahievran.edu.tr

Hamza Çalışıcı

Ondokuz Mayis University, Faculty of Education, Department of Mathematics, Kurupelit/Atakum, Turkey

E-mail address: hcalisici@omu.edu.tr

Ergül Türkmen

Amasya University, Faculty of Art and Science, Department of Mathematics, İpekköy/Amasya,Turkey

E-mail address: ergulturkmen@hotmail.com 